BIOMEDICA

Vol. 3 No. $4-1983$

INFORME DE CASOS

\title{
PROTOTECOSIS
}

\section{INFORME DE TRES CASOS}

\author{
MIGUEL GUZMAN, ${ }^{*}$ GERMAN RAMIREZ, ${ }^{\star *}$ BERNARDO BUITRAGO .**
}

\begin{abstract}
Se presentan tres casos clínicos de prototecosis vistos y estudiados en el Instituto Nacional de Salud. Uno corresponde a la forma tegumentaria y los otros dos a formas de bursitis del olécranon.
\end{abstract}

\section{Se discuten los aspectos biológicos, clínicos y de laboratorio de esta rara entidad causada por algas.}

\section{INTRODUCCION}

Los cuadros patológicos causados en el hombre por algas son en extremo raros; desde el caso de Davies (1) en 1962 ha existido interés por este tipo de patología. El presente informe destaca 3 cuadros vistos y estudiados en nuestra institución.

\section{Casos clinicos}

\section{Caso No. 1}

Hombre de 40 años de edad. economista, natural de Medellín y procedente de Bogotá, casado. Consulta por tumefacción en el codo derecho, dolor intenso y sensación de ardor, con evolución de 8 días. Al examen físico se encuentra el paciente de complexión atlética en buen estado general; presenta en el codo derecho una tumefacción localizada sobre el olécranon, renitente, ligeramente dolorosa a la presión que limita discretamente el movimiento de flexión del antebrazo (Fig. No. 1). Refiere el paciente que días anteriores presentó ligeras grietas cutáneas por sequedad de la piel, habiéndose aplicado una crema emoliente; como antecedente recuerda haber jugado tenis de mesa dos días antes de iniciarse el proceso y haber asistido a un paseo de campo en donde posiblemente apoyó el codo en el prado; refiere que el proceso se inició en forma súbita; inicialmente fue examinado por un médico quien consideró una posible infección estreptocócica habiendo recibido penicilina sin que este tratamiento tuviese efecto alguno; por tal razón, consultó nuevamente al INS. La impresión diagnóstica inicial al ser visto por uno de los autores fue de una bursitis del olécranon, inmediatamente se hizo una punción extrayéndose aproximadamente $15 \mathrm{ml}$ de un líquido amarillo verdoso, inodoro. Las preparaciones en fresco bajo campo claro con objetivo $40 \mathrm{x}$ mostraron abundantes polimorfonucleares y unos elementos de morfología variable con estructuras internas similares a esporas que fueron interpretadas como células de Prototheca sp. (Fig. No. 2); igualmente se hicieron preparaciones para coloraciones de plata-metenamina que mostraron estructuras celulares identificadas como Prototheca (Figura No. 3). Se hicieron también cultivos

\footnotetext{
* Jefe, Sección Diagnóstico, Investigación y Referencia - Instituto Nacional de Salud. Profesor Asociado, Facultad de Medicina - Universidad Nacional - Bogotá, Colombia.

** Jefe, Sección de Control Biológico, Instituto Nacional de Salud, Bogotá, Colombia.

*** Jefe, Grupo de Patología, Instituto Nacional de Salud - Bogotá, Colombia.
} 


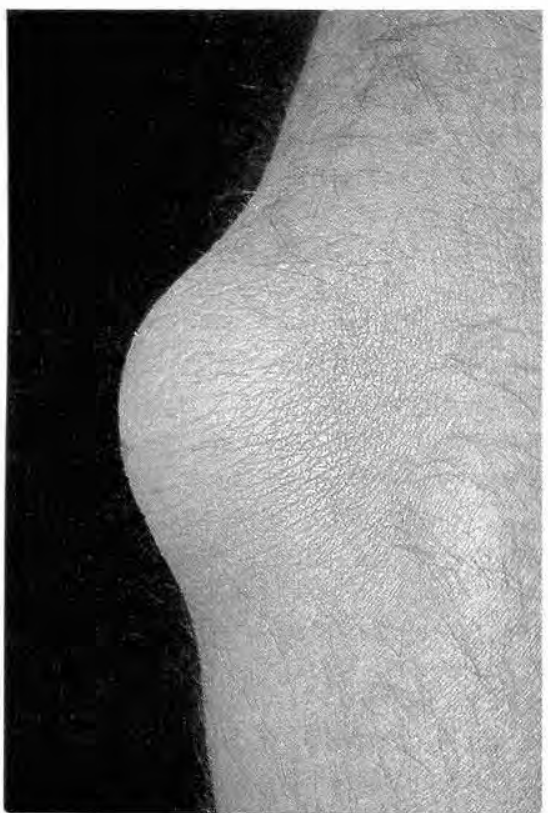

Figura No. 1. Tumefacción del codo por un proceso de bursitis, de cuyo líquido se aisló Prototheca sp.

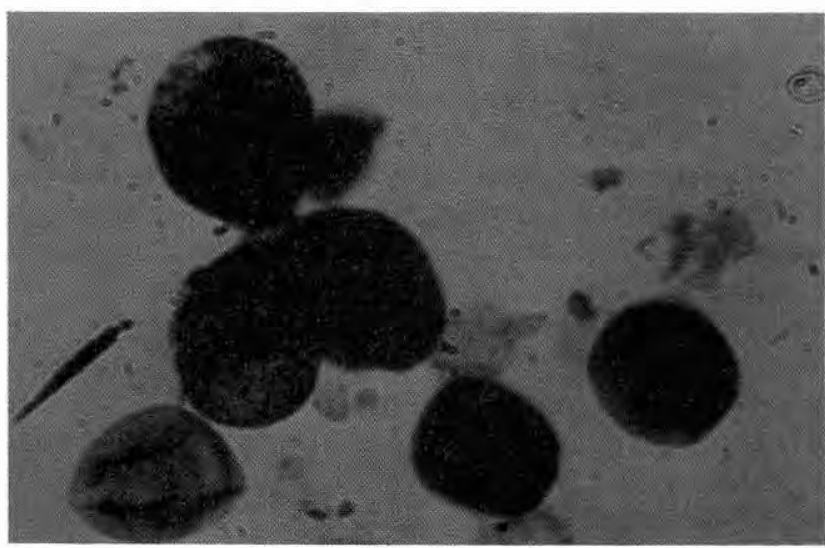

Figura No. 3. Células de Prototheca coloreadas con plata-metenamina. Corresponden al caso No. 1.

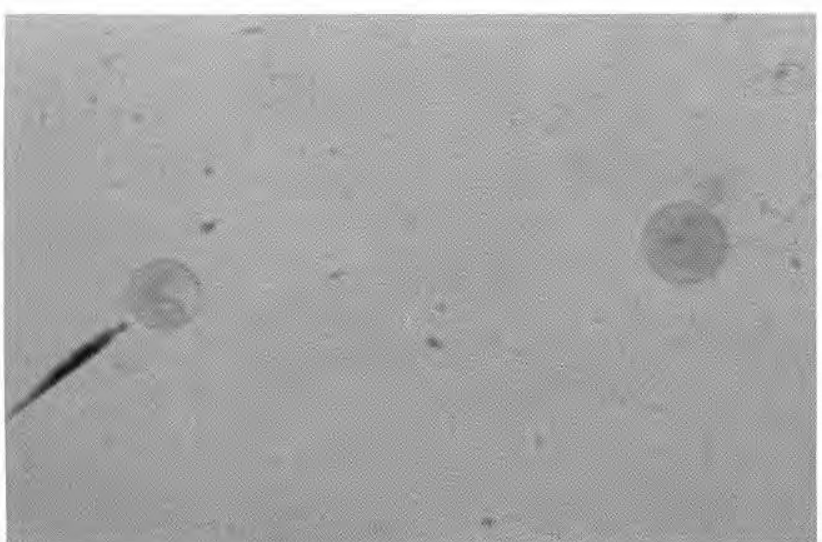

Figura No. 2. Células de Prototheca, del líquido obtenido de la lesión. Preparación en fresco 1.250 x.

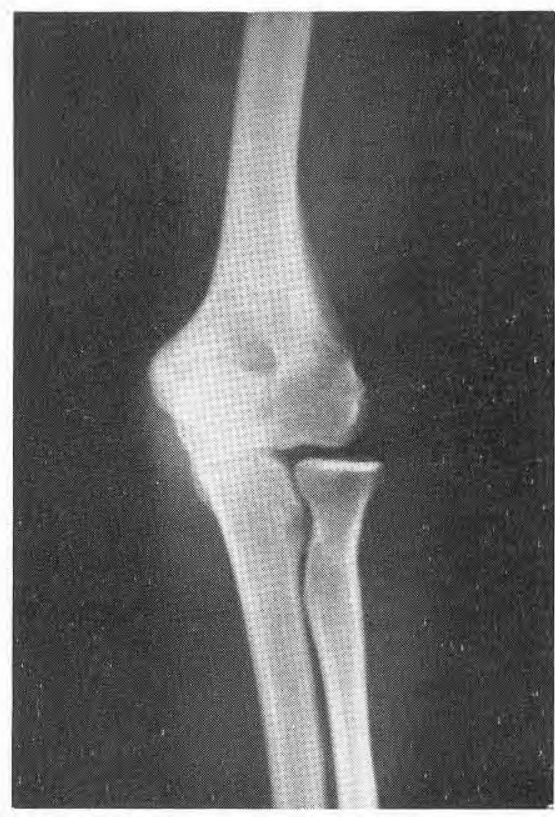

Figura No. 4. Radiografía de codo del caso No. I. No se observan alteraciones óseas ni articulares. 
en medio líquido y en Sabouraud glucosado, habiéndose obtenido crecimiento en medio líquido el cual mostró las mismas estructuras celulares vistas en fresco pero fallándose persistentemente en obtener sub-cultivos en medios sólidos. Al paciente se le ordenaron estudios radiológicos que no mostraron compromiso óseo, ni articular (Fig. No. 4). Igualmente se le ordenó un estudio inmunológico completo de tipo humoral y celular el cual no mostró alteración alguna. El paciente se recuperó gradualmente y en término de un mes la lesión desapareció completamente y espontaneamente sin dejar secuela. El diagnóstico final fue bursitis del olécranon por Prototheca sp.

\section{Caso No. 2}

Mujer de 32 años, natural y procedente de Tai-pei, ama de casa, consulta por lesión de uña del dedo índice derecho de aproximadamente 6 semanas de evolución. Refiere que el proceso se inició luego de un pequeño traumatismo local durante a rreglo de uñas en una sala de belleza, el cual se repitió el mismo día al pelar mariscos. Al examen físico se encuentra una lesión úlcerocostrosa del tejido periungueal que compromete la uña y que exuda material purulento. Al ser vista había recibido diferentes tratamientos locales con cortiscosteroides sin mejoría alguna (Fig. No. 5). Debido a la severidad de la lesión se hizo resección quirúrgica de ésta y la pieza

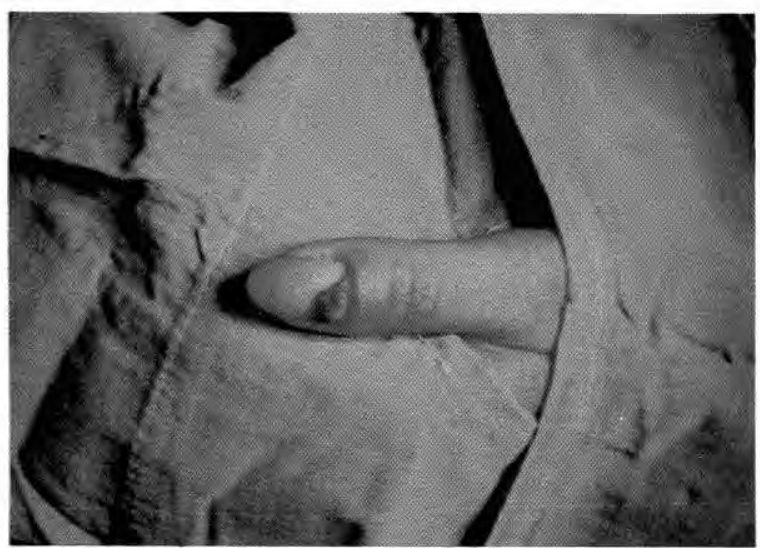

Figura No. 5. Lesión periungeal, caso No. 2. se estudió microscópicamente. El estudio histopatológico incluyó cortes y tinciones con Hematoxilina-Eosina, PASH, Azul-Alciano, Grocott y Orceina-Giemsa. Se observó una capa córnea hiperqueratótica con zonas de aspecto esponjoso o necrótico. La epidermis era acantótica con alargamiento de las crestas interpapilares con edema intesticial y exocitosis de polinucleares que constituían abscesos en el corion subyacente; en medio de este material se observan huecos muy vecinos entre sí, que con las tinciones para mucopolisacáridos aparecen ocupados por esporas de dimensiones variables, que constituyen mórulas de unas 15 micras de diámetro; en otros sitios alternan con estructuras de tamaño muy superior cuya envoltura se tiñe con PAS y que parece corresponder a tecas bi, tri, o multiesporuladas. Esta morfología no corresponde a hongos, sino que sugiere la presencia de alga aclórica posiblemente del género Prototheca, (Fig. No. 6).

El paciente retornó a Tai-Pei con el diagnóstico de prototecosis ungueal habiéndo recibido tratamiento con derivados del Imidazol recuperándose totalmente.

\section{Caso No. 3}

Hombre de 48 años, procedente de Bogotá, profesión médico. Refiere que hace tres días permaneció unos minutos acostado en el pasto apoyado sobre el codo izquierdo, notando posteriormente que el césped estaba húmedo; 6 horas más tarde sintió

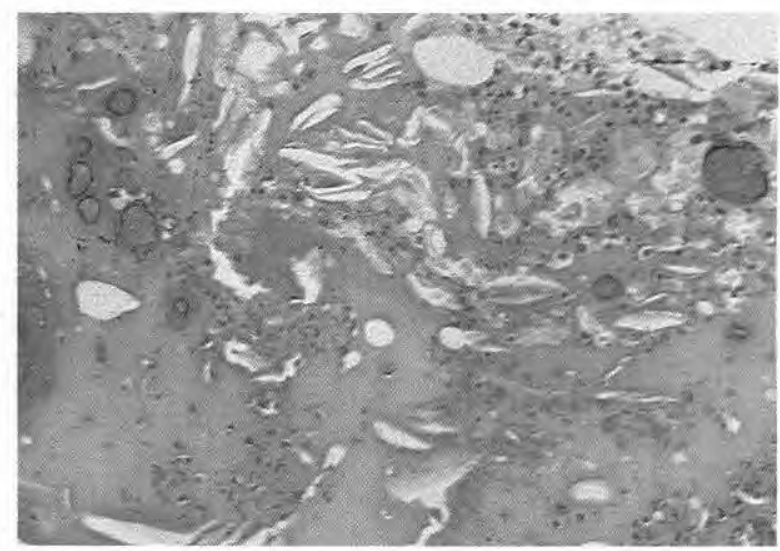

Figura No. 6. Corte histológico de la lesión del caso No. 2. Obsérvese las células de Prototheca. 


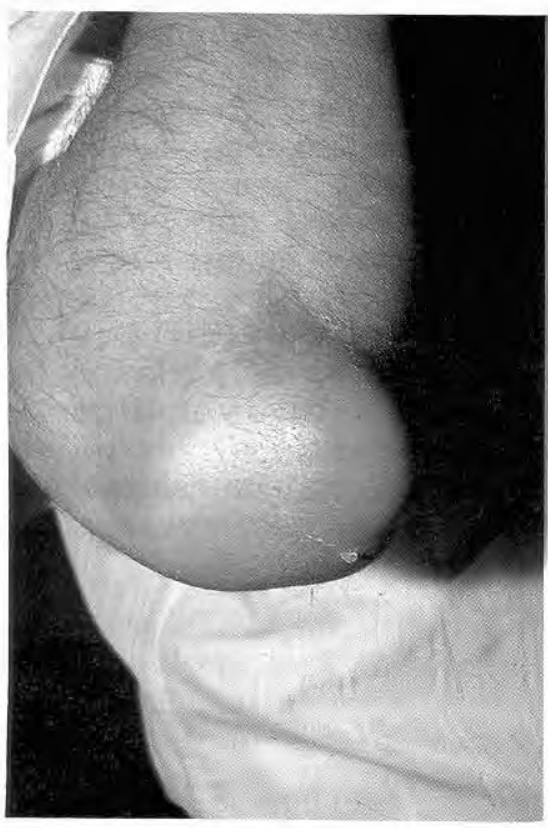

Figura No. 7. Bursitis del codo, del caso No. 3. Nótese la severidad del proceso inflamatorio.

dolor a la presión en el codo y tres horas después presentaba una tumefacción dolorosa enrojecida con piel brillante de un diámetro de 3 a $4 \mathrm{~cm}$ (Fig. No. 7). Al día siguiente debido a la disminución funcional de codo y al dolor optó por puncionar la tumefacción saliendo un líquido amarillo transparente. A los dos días. la lesión del codo se había llenado nuevamente por lo que consultó al INS. Se hizo otra punción obteniéndose unos $10 \mathrm{ml}$ del líquido amarillo inodoro y transparente que se utilizó para exámenes posteriores. La lesión volvió a llenarse y por tercera vez se puncionó, obteniéndose nuevamente el líquido amarillento transparente; luego de esta última aspiración, en la cual se obtuvieron $3 \mathrm{ml}$, la lesión comenzó a disminuir sin más tratamiento que calor local.

Dos meses antes había presentado un traumatismo en el codo izquierdo produciendo una gran zona de escoriación que se convirtió posteriormente en costra la cual cayó dejando una zona enrojecida. En el momento de la enfermedad actual la zona permanecía enrojecida y descamativa.
El examen directo del líquido mostró gran cantidad de polimorfonucleares y entre éllos estructuras redondeadas semejantes a células de Prototheca sp., las preparaciones teñidas con Plata-Metenamina mostraron sin ninguna duda las formas endospuruladas de Prototheca. (Fig. No. 8). Ios cultivos realizados sobre Sabouraud glucosado y medios líquidos mostraron crecimiento en 48 horas (Fig. No. 9); en los medios sólidos se

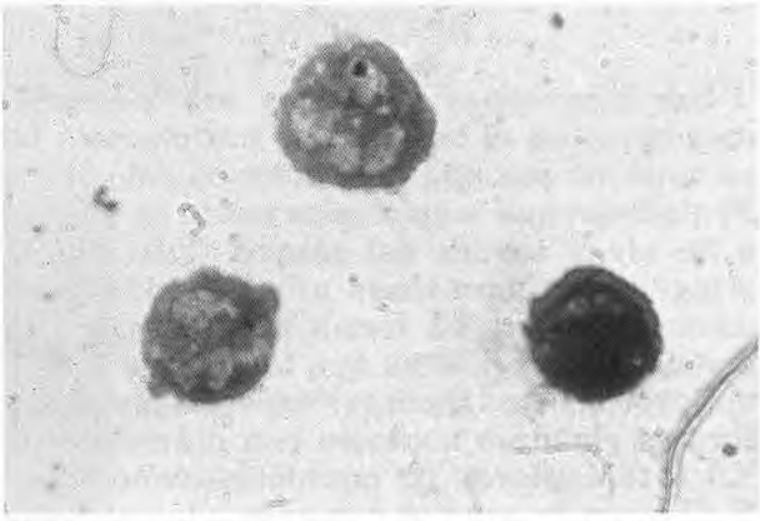

Figura No. 8. Células de Prototheca, coloreadas con plata-metenamina, a partir del líquido extraído de la lesión del caso No. 3.

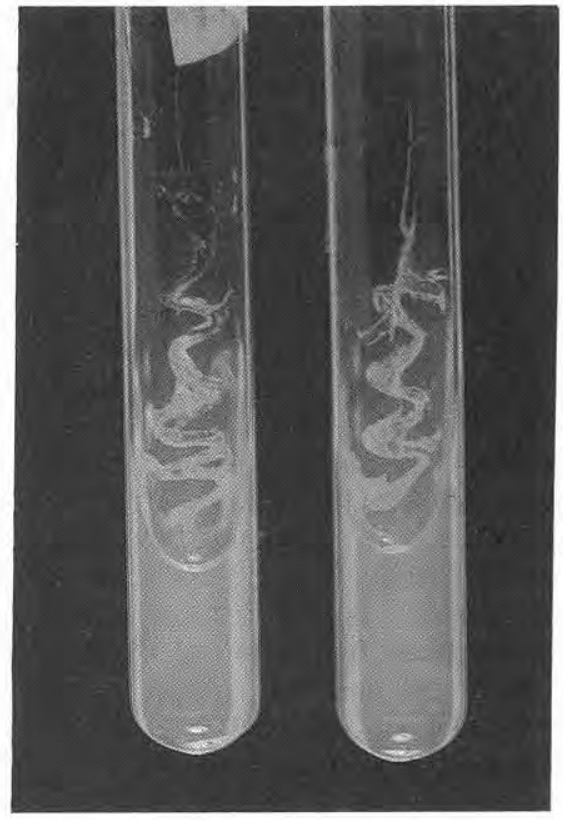

Figura No. 9. Cultivo de Prototheca sp. del caso No. 3, después de 48 horas de incubación en medio de Sabouraud glucosado. 
observaron colonias compactas tipo levaduras que al examen directo y coloración de Gram corresponden a las mismas estructuras observadas en el examen directo. Es de anotar que estas estructuras no toman el Gram.

Cuatro semanas después de iniciado el proceso, se presentaba una remisión casi total de la lesión. Como tratamiento solo se utilizó calor local en forma de fomento.

\section{DISCUSION}

Las infecciones por algas son entidades muy raras en el hombre. La prototecosis (2) es causada por una alga aclórica del género Prototheca que morfológicamente es similar a las algas verdes del género Chlorella. El microorganismo tiene una reproducción asexual que en su forma madura muestra divisiones irregulares que forman de 2 a 20 endosporas. Las distintas especies muestran formas globosas u ovales con diámetros de 1,3 a 13,4 micras. El microorganismo crece fácilmente en medios comunes que carezcan de cicloheximida; en 48 horas hay un buen crecimiento dando colonias húmedas, blancas o canela pálidas, similares a colonias de levaduras; la temperatura óptima es de $25-30^{\circ} \mathrm{C}$; algunas especies no crecen a $37^{\circ} \mathrm{C}$. Dentro del género Prototheca hay por lo menos tres especies que se han implicado en cuadros humanos: $P$. stagnora, $P$. wickerhamii y $P$. zopfii. $(2,3,4)$.

Los microorganismos del género Prototheca causan infecciones tanto en el hombre como en los animales. $(3,5,6,7)$. Los cuadros causados en el hombre pueden dividirse en tres formas clínicas: formas que afectan la piel y el tejido celular subcutáneo que generalmente se presentan en zonas expuestas, son progresivas, lentas y con poca tendencia hacia la curación espontánea (2). Otra forma es la bursitis del olécranon (8); en los casos informados en la literatura médica la lesión aparece frecuentemente sin que haya traumas penetrantes del codo; la tercera forma es muy rara y corresponde a la forma diseminada. (9). El número de casos de prototecosis humana confirmados no pasa, seguramente, de los treinta (10) en el mundo; en la revisión de Kaplan (2) aparecen 18 casos: 8 bursitis,
9 de piel y tejido celular subcutáneo y 1 diseminado. Se conoce muy poco de su epidemiología, si bien está frecuentemente presente como saprofito en lama de árboles, aguas de lagos, plantas, aguas marinas y aguas de desecho; no se conoce mucho acerca de las condiciones en que se expresa su patogenicidad, algunos autores la consideran como un oportunista (2). El diagnóstico se basa en el hallazgo del microorganismo, su cultivo y tipificación bioquímica, su presencia en los tejidos evidenciada por histología con coloraciones específicas, y por inmunofluorescencia directa (2).

En cuanto a tratamiento se han ensayado ioduros, Anfotericina B y derivados de imidazol con resultados variables. Algunos casos curan espontáneamente otros necesitan resección quirurgica. Los casos aquí reseñados son, posiblemente, los primeros informados en Colombia; el primero corresponde a la forma clínica de piel y tejido celular subcutáneo curado por resección quirúrgica y los otros dos a una forma de bursitis del olécranon con curación espontánea; en ninguno de ellos se encontró una condición de base que pudiera explicar el proceso y, con excepción del primer caso, en que hubo un claro antecedente de trauma y penetración, los dos casos de bursitis no tienen antecedente de trauma penetrante, tal como ha ocurrido en los casos hasta ahora informados (6). Como caso curioso de coincidencia, sin implicaciones de tipo epidemiológico de ninguna naturaleza, se menciona el hecho de que los dos casos de bursitis ocurrieron en personal de una misma institución, presentándose el primero de éllos en 1981 y el segundo en 1984 en el médico que atendió el primer caso.

\section{SUMMARY}

Three clinical cases of Protothecosis seen and studied in our Institute are presented. One of them correspond to the skin and soft tissue type and the other two correspond to bursitis of the olecranon. None had any demostrable underlying disease.

The biological, clinical and laboratory aspects of this rare disease caused by algae are discussed. 


\section{BIBLIOGRAFIA}

1. Davies RR, Spencer H. Wakelin PO. A case of human protothecosis. Trans. Royal Soc. Trop. Med. Hy. 1964; 58: 448.

2. Sudman MS. Prothecosis. A critical review. Am J. Clin. Pathol. 1974; 61: 10.

3. Kaplan W. Protothecosis and infeccions caused by morphological similar green algae. Proceedings of the IV International Conference on Mycosis. Pag. 218. Pan American Health Organization. 1978.

4. Davies RR, Wilkinson JL. Human protothecosis: supplementary studies. Ann Trop. Med. Parasitol. 1967: 61: 112 .

5. Frank N. et al. Protoheca, a cause of bovine mastitis Am J. Vet. Res. 1969; 30: 1985.
6. Holscher MA. et al. Disseminated canine protothecosis: a case report. J. Am An. Hos. Assoc. $1976 ; 12: 49$.

7. Kaplan W. Protohecosis in a cat: first recorded case, sabouraudia. 1976; 14: 281.

8. Nosanchuk JS. Greenberg RD. - Protothecosis of the Olecranon Bursa caused by achloric algae. Am. J. Clin. Pathol. 1973; 59: 567.

9. Cox et al. Protothecosis: a case of disseminated algal infection. Lancet. 1974; August 17: 379.

10. Connor DH, Gibson DW, W Zincter A. - Diagnostic Features of Three unusual infections: Micronemiasis, Pheomycotic cyst and Protothecosis; chapter 13 , pp. 205. Current topics in inflammation and infection. International Academy of Pathology Monograph. Williams - Wilkins Editors. Baltimore/London, 1982. 